\title{
Design and Study on Mechanical Properties of Aircraft Wing Lift Simulation Device
}

\author{
Qing-Bo DOU ${ }^{1, \star}$, Xiao-Chuan LIU ${ }^{2}$ and Rang-Ke MOU ${ }^{2}$ \\ ${ }^{1}$ School of Aeronautics, Northwestern Polytechnical University, Xi'an 710012, China \\ ${ }^{2}$ AVIC Aircraft Strength Research Institute, Xi'an 710065, China \\ *douqb527@163.com
}

Keywords: Wing lift, Mechanical properties, Test, Loading criteria, Landing.

\begin{abstract}
Full scale aircraft drop test was an effective method to assess dynamic loads and response in indoor environment. Wing lift simulation with drop testing machine is to impose a dynamic load equaling the landing weight on the aircraft, so as to completely simulate the landing condition. The choice of loading mode and amplitude is the critical factor for drop tests of aircraft. This paper proposes a wing-lift loading system suitable for full scale aircraft drop test. The principle of this loading system is first introduced. The proposed wing lift loading system is validated using quasi-static and dynamic tests. Wing lift loading mode and criterion are proposed based on the experimental results. Finally, the proposed loading method and system is applied to a full scale aircraft drop test, experimental results confirm the validity of the feasibility of the loading method and the proposed wing lift simulation system.
\end{abstract}

\section{Submitting the Manuscript}

Landing load tests are usually carried out during the development of the aircraft by landing gear tests [1-2]. Different from land-based aircraft, sinking speed of carrier-based aircraft can achieve 7 $\mathrm{m} / \mathrm{s}$ or even higher, which is more than twice as large as common aircraft, and the landing gear absorb energy is 4 6 times[3-6] than normal plane. In order to verify the dynamic performance of aircraft under landing load, such as cushioning performance of the landing gear, the dynamic response of the aircraft structure, the reliability of the important functional components and overload of the driver, occupant, and so on, the carrier aircraft generally through full scale aircraft drop test In the laboratory environment to assess the aircraft landing load and the important equipment dynamic response caused by landing load. [7-9].

Carrier-based aircraft landing with a certain heading speed landing, and the wing lift has been imposed on the aircraft, ready to take off when landing failed. In order to simulate the shipboard loading in a laboratory environment, it is necessary to apply the wing lift by means of a specific device to evaluate the landing load and structural response of the aircraft. How to simulate the lift of the wings in full scale aircraft drop test of the carrier-based aircraft, foreign public information rarely reported. The landing load of carrier-based aircraft is more common in theoretical simulation [10-12], while the landing-wing lift simulation is more common in landing gear drop test [13]. According to the aircraft design and test specification, Yao Niankui put forward the requirements and analysis methods of the full scale aircraft drop test of the aircraft [14-15]. The full scale aircraft drop test has not been carried out in China. And also no studies on the lift loading method and the lift simulator for the full scale aircraft drop test.

This paper introduces a kind of wing lift loading method and loading criteria apply to full scale aircraft drop test. A wing lift simulator based on air medium is designed and validated. And applies the device and the method to a full scale aircraft drop test of a certain type aircraft. The experiment results show that the device and the wing lift loading method can effectively simulate the wing lift in the full scale aircraft drop test. 


\section{Loading Method of Full Scale Aircraft Drop Test \\ Method of Wing Lift Loading}

Full scale aircraft drop test of carrier-based aircraft is a large-scale dynamic test, which is generally carried out in a dedicated test platform. The text simulated the aircraft landing attitude, such as vertical velocity, horizontal velocity and the wing lift in the process of landing. Through the multichannel and multi-physical testing system, the dynamic load and response of the body, landing gear and other structural parts during the test are recorded. Block diagram of the full scale aircraft drop test shown in Figure 1.

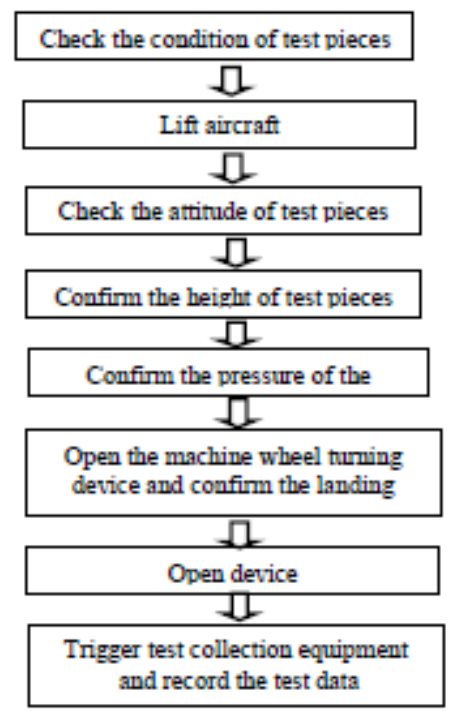

Figure 1. Full scale aircraft drop test flow diagram

When the full scale aircraft drop test is performed, the aircraft is raised to the predetermined height by the lifting device. And aircraft wheel reverse rotate to simulate the aircraft landing speed by the wheel rotate equipment, when aircraft line speed reaches a predetermined speed, then sudden release the aircraft, and the aircraft free fall. When the aircraft touches the ground force measuring platform and simultaneously applies the wing lift of the size equal to the weight of the airplane. The test data acquisition system is triggered before the plane touched the force measuring platform, and the time history curve of each sensor is recorded after the aircraft touched the platform.

The wing lift simulation system consists of a bearing frame, a wing lift simulator, and an adjustable tension rod. Figure 2 shows the schematic diagram of the lift loading method. Upper part of the wing-lift simulator is connected with the load-bearing frame by the soft-connection, which avoids the additional moment along the course of the wing-lift simulator under the heading load when the aircraft touches the platform. Lower part of the wing lift simulator is connected with the wing pallets through the adjustable rod, and the wing lift is transmitted to the two side wings by the wing pallets to simulate the wing lift simulation in the laboratory environment.

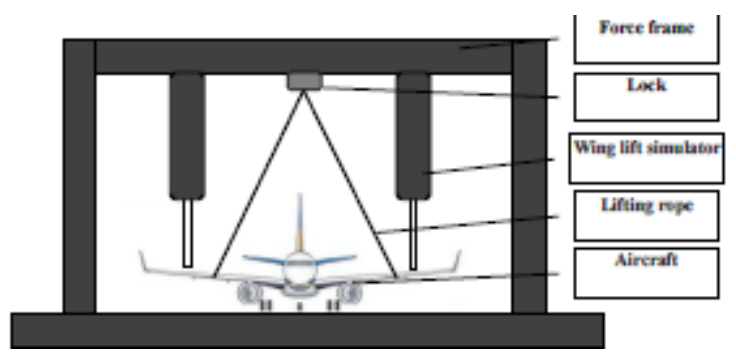

Figure 2. The wing lift simulation diagram of full scale aircraft drop test 


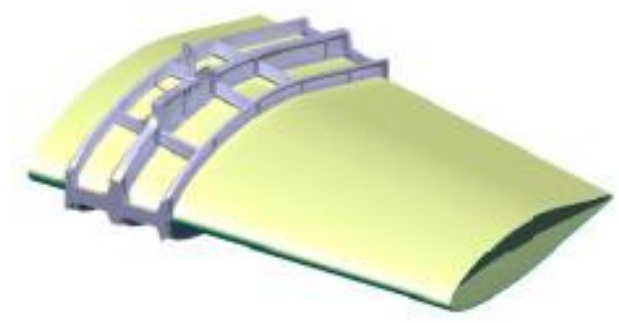

Figure 3. The wing loading device design diagram

\section{Wing Lift Loading Criteria and Methods}

As the key technology of the Full scale aircraft drop test, lift simulation of the wing has definite requirements on the size, the working time and the attitude of the aircraft. First, the lift of the wing should be kept constant during the compression process of the aircraft landing gear; Secondly, the application of wing lift force should not affect the instantaneous vertical velocity of aircraft contact force platform; Third, It should not affect the attitude of the aircraft landing after apply the lift of the wing in the process of aircraft dropping. In view of the requirements of the above three aspects, the plane left and right wings of the plane are respectively provided with a constant load of $1 / 2$ aircraft landing gravity by adopting the two air operated cylinder with gas storage function. The aerodynamic cylinder with the function of gas storage is called the wing lift simulation device. Gas cylinders with gas cylinders are used for two aspects, First of all, due to the pneumatic cylinder with a faster load output response time relative to other devices, it can be more quickly when the impact load in the simulation of the impact load of the ship. Secondly, the output load and the cylinder internal gas pressure into a linear relationship in the case of a gas cylinder piston area, it is more conducive to the output load control. Theoretically, the change in the gas pressure on the piston's internal area of the air cylinder remains constant, ensuring that the simulated lift provided by the wing lift simulator remains constant.

The wing lift load point is in the vertical section which is perpendicular to the direction of the fuselage through the center of gravity of the fuselage. And the wing lift simulation device is perpendicular to the ground installation, and the wing is connected with a double hinge, release rotational degrees of freedom of the vertical rotation, and the effect of simulating wing lift will not affect the aircraft landing attitude.

To control the lift force of the wing, when the carrier-based aircraft drop to the force platform, the lift force of the wing is applied suddenly, so as to the effect of simulating wing lift will not change the size of the sinking velocity of the test piece.

\section{Working Principle and Structure Design of Lift Simulation Device Structure Principle}

The lift simulation device is realized in the form of a pneumatic lifting device, there are two advantages using gas cylinder as lift simulation device. On the one hand, under the certain circumstances of piston area and gas storage room capacity, we can adjust the size of the simulated lift by changing the pressure of the gas storage room, it can meet the simulation needs of different sizes of lift. It has better general performance. On the other hand, under the certain circumstances of the gas pressure in the cylinder is relatively constant, the load of the cylinder piston output is a certain value, and the wing lift of the aircraft landing gear compression section should be relatively constant. Wing lift simulation device structure diagram shown in diagram 4. 


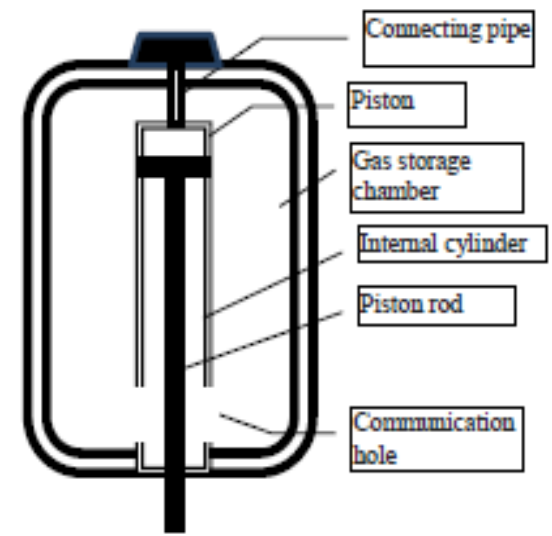

Figure 4. Wing lift simulation device structure diagram

Lift simulation device is divided into storage room, internal cylinder, piston rod. The piston rod is connected to the wings on the outside of the cylinder and is connected internally with the piston to compress the air through the piston to provide wing lift. Top end of the internal cylinder is held in communication with the atmosphere by the external connecting pipe, thus ensuring that the internal piston can move along the internal cylinder under tension. The gas storage chamber and the internal cylinder are communicated through the internal communication hole so that the internal pressure of the reservoir chamber is made as small as possible due to the movement of the piston in the cylinder so as to ensure that the lift of the simulated wing output by the piston rod is kept substantially constant.

\section{Structural Parameters of Lift Simulation Device}

Assuming that the initial pressure of the lifting simulation device is $\mathrm{P} 0$, the initial volume is $\mathrm{V} 0$ (including the volume of the gas storage chamber and the volume of the lower part of the piston).During the piston motion, the effective volume of the system is reduced. Assuming that the system volume loss is $\Delta \mathrm{V}$ in the end of compression stroke, and the system pressure is $\mathrm{P} 1$ at this time, the storage volume is $\mathrm{V} 0-\mathrm{V}$. According to the second law of thermodynamics, In a certain area of the piston, neglecting the system friction and dynamic inertia force of piston and piston rod, the output load of the system is that the simulation of the wing lift is only related to the system pressure from the formula (1) and (2).

$$
\begin{aligned}
& P_{0} V_{0}^{\gamma}=P_{1}\left(V_{0}-\Delta V\right)^{\gamma} \\
& \frac{P_{1}}{P_{0}}=\left(\frac{V_{0}}{V_{0}-\Delta V}\right)^{\gamma}=\left(\frac{1}{1-\frac{\Delta V}{V_{0}}}\right)^{\gamma}
\end{aligned}
$$

Where $\gamma$ is the gas changeability index, $\Delta \mathrm{Vis}$ related to piston area $\mathrm{A}$ and displacement $\mathrm{L}$, $\Delta \mathrm{V}=\mathrm{A} \times \mathrm{L}$. Output simulation of wing lift $\mathrm{T}=\mathrm{P} \times \mathrm{A}$, to keep the output of simulation approximately constant (such as the maximum variation not more than 5\%), which requires the maximum relative changes in the amount of pressure is not more than 5\%. From (2) can be known, only to meet the relative change in the system volume is not more than $4.3 \%(\gamma=1.1)$. By designing the piston area of the cylinder and the volume of the gas storage chamber reasonably, the design requirements of the output lift can be satisfied. The size of simulative wing lift can be obtained by formula (3).

$$
F=P \times \pi\left(R_{1}^{2}-R_{2}^{2}\right)
$$

In the formula, $\mathrm{P}$ is wing lift simulator pressure, $\mathrm{R}_{1}$ is wing lift simulator piston radius, and $\mathrm{R}_{2}$ is piston rod radius.

Take the maximum output payload of $160 \mathrm{kN}$ as an example. According to the above design criteria, assuming the displacement of the piston is $1 \mathrm{~m}$, determine the main reference design parameters in Table 1, the theoretical pressure-load curve shown in Figure 5. 
Table1. The main design parameters

\begin{tabular}{|c|c|c|c|c|c|c|}
\hline Name & $\mathrm{P}_{\max }$ & $\mathrm{V}_{0}$ & $\mathrm{R}_{1}$ & $\mathrm{R}_{2}$ & $\mathrm{~L}_{\max }$ & error \\
\hline Unit & $\mathrm{MPa}$ & $\mathrm{m}^{3}$ & $\mathrm{~m}$ & $\mathrm{~m}$ & $\mathrm{~m}$ & $\%$ \\
\hline Design value & 6.06 & 1 & 0.1 & 0.04 & 1 & 2.99 \\
\hline
\end{tabular}

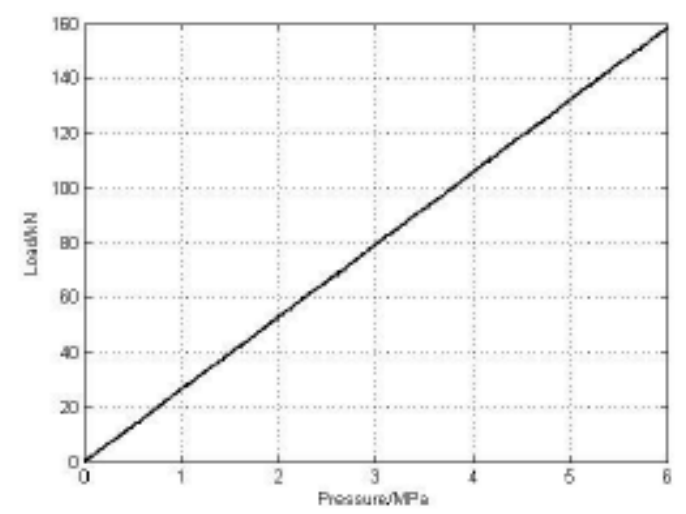

Figure 5. The theory curve of pressure-load

\section{Mechanical Performance Verification of Wing Lift Simulation Device}

\section{Static Mechanical Performance Verification}

The static pressure test of a single wing lift simulator was carried out to verify the correspondence between the pressure and the output load of the wing lift simulator under static conditions.

Figure 6 shows a static verification test of the wing lift simulator. Upper end of the wing lift simulator is mounted on the load gantry frame, and the load sensor is connected to the lower end of the piston rod. And the additional weight is $12000 \mathrm{~kg}$. According to the gas distribution table shown in Figure 5, the internal gas pressure of the wing lift simulation device is adjusted. The internal gas pressure and output load of the device are collected by installing the pressure sensor in the lift simulation device and the load sensor on the lower end of the piston rod during the experiment. In the static case, the relationship between the input pressure and the output load of the system is checked to verify the compliance with the theoretical calculation.
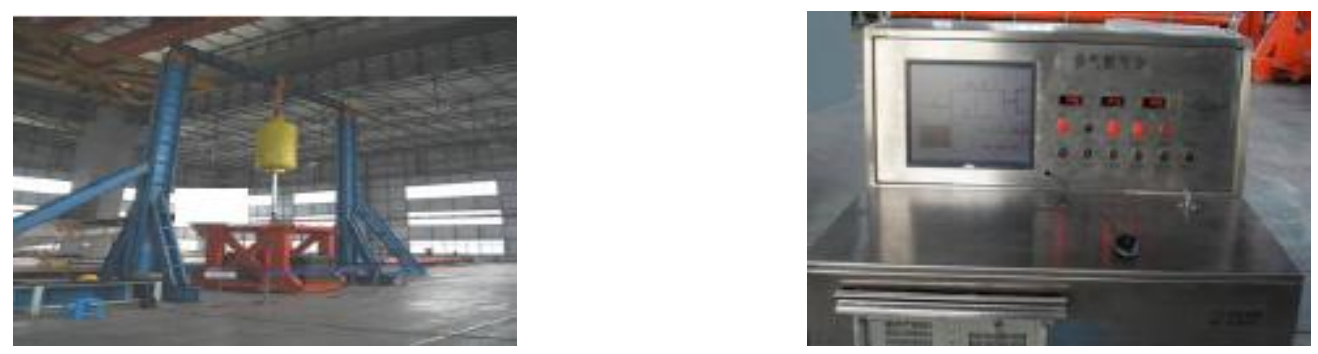

Figure 6. Wing lift simulation device static debugging Figure 7. Gas pressure regulating equipment

Real-time pressure-load curve of the wing lift simulator is obtained. Correlation table of a typical data point design pressure-load data and test data comparison in the process of static verification of the wing lift simulation device is shown in table2. The pressure-load test curve and the design theory of the wing lift simulation device are shown in figure 8.

Table 2. Measured and theoretical value comparison

\begin{tabular}{|c|c|c|c|c|}
\hline Air pressure (MPa) & 1 & 2 & 3 & 4 \\
\hline Theoretical load (kN) & 26.01 & 52.40 & 78.79 & 105.20 \\
\hline Load (kN) & 24.59 & 51.08 & 77.89 & 104.72 \\
\hline Error (\%) & $5.45 \%$ & $2.52 \%$ & $1.14 \%$ & $0.45 \%$ \\
\hline
\end{tabular}




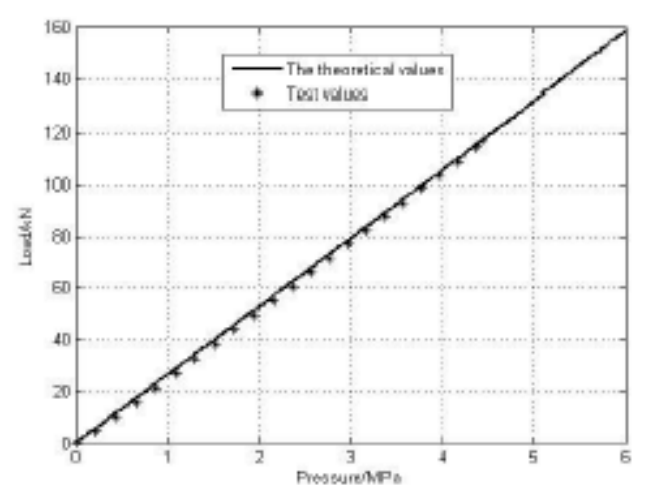

Figure 8. Measured and theoretical pressure-load curve

It can be seen from Table 2 that the measured output load of the wing lift simulator is corresponding to the calculated theoretical value when the pressure of the air chamber is small, and gradually decreases as the pressure in the chamber increases.

The reason for this result is mainly due to two points: 1 , the piston of wing lift simulation device need to overcome static friction gradually between the piston ring and the cylinder wall when the air pressure is small, and the friction is opposite to the direction of the piston movement. 2, weight lifting point and lifting direction of piston rod are not in a vertical line strictly, and stress direction coordinated gradually in the process of ascension, tending to vertical direction.

\section{Dynamic Mechanical Performance Verify}

After the static verification, the wing lift simulator is dynamically checked to verify whether the performance characteristics of the wing under the dynamic impact conditions meet the requirements of the wing lift loading.

The procedure of dynamic verification test is as follows, installing two wing lift simulation device on gantry frame, it is connected with a certain weight mass block in the lower end of wing lift simulation device. The mass block is connected with the electromagnetic release lock, and the inside of the wing lift simulator system is pressurized, the system pressure reaches the specified value, the piston is located in the top of the internal cylinder. Then electromagnetic release lock suddenly release the mass, the mass under the action of gravity to produce a certain initial speed, mass down in the process of movement. During the downward movement of the mass block, the piston rod of the wing-lift simulator is suddenly tensioned to generate an impact load and to drive the piston downwards. Test system records the time history data of the load cell and the pressure sensor, and analyzes the dynamic characteristics of the wing lift simulator based on the recorded data. The build schematic of dynamic verification test system shown in figure 9.

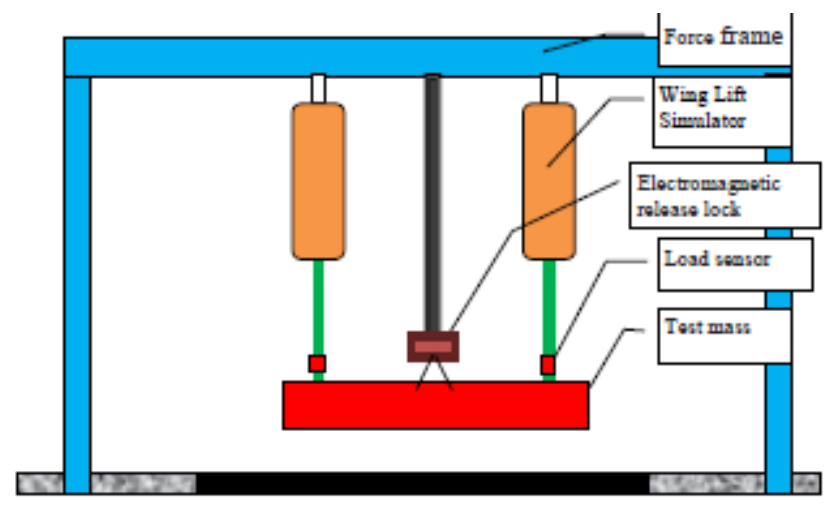

Figure 9. Dynamic debugging test schematic

The contrast curve between internal gas pressure initial pressure of wing lift simulation device and measured value of gas pressure in the internal device is shown in figure 10. Comparison of 
simulated lift test curve of the two wing lift simulation device and the theoretical load curve are shown in figure 11.

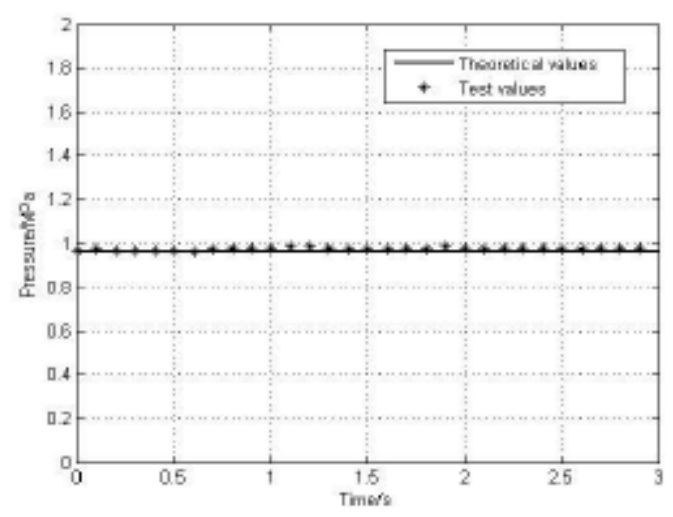

Figure 10. Test and theoretical comparison of gas pressure

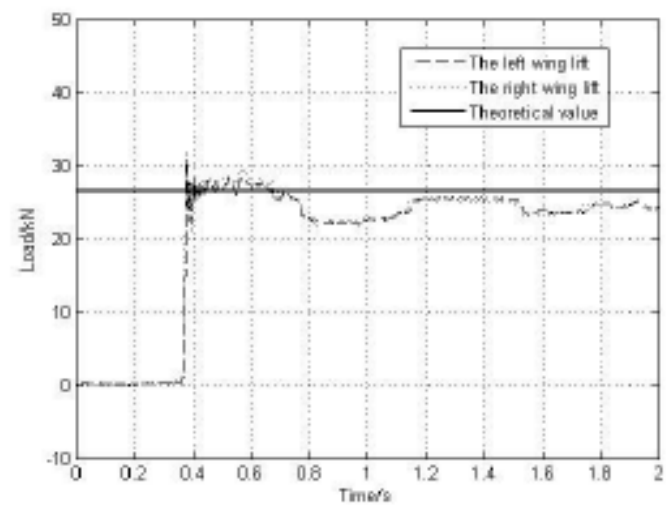

Figure 11. Test and theoretical values contrast curve

From the test data, we can see that the change between internal gas pressure and initial pressure during dynamic drop test is less than $2 \%$. The measured output load is slightly greater than the theoretical output load in the stage of the mass block declining. This is due to the dynamic process of falling, there is dynamic friction between the piston and cylinder wall, at the same time, due to the inertia load of the piston and piston rod under the impact load, measured load is slightly larger than the theoretical value in the combined effect of friction and inertia load. In the reverse stroke, because the friction between cylinder piston and the seal ring is opposite, and the simulated lift force appears step decreasing. In the case of a certain height, the step value is about $2 \mathrm{kN}$. At the same time, it can be seen from the measured curve of the load that the simulated lift force is imitated at the moment of impact loading due to the sudden output of the simulated lift force in the wing lift simulator, and the spike load should be reduced and avoided in the actual data processing.

For the reason of the peak load caused by the lift of the wing, the mass block is used as the research object and the momentum theorem is applied.

$$
\begin{gathered}
(T-M g) \Delta t=M V-0 \\
T=M g+\frac{M V}{\Delta t}
\end{gathered}
$$

In the formula, $\mathrm{T}$ is the wing lift, $\mathrm{M}$ is the weight of load, $\mathrm{V}$ is the speed of the lift load when the lift load, and $\Delta t$ is the action time of lift loading. According to the formula (5), to reduce the impact peak load, we only need to increase the action time when the lift is loaded suddenly. We can reduce the peak load by using proper buffer device to increase the wing lift in Engineering. In test data processing, through simulation of wing lift in landing gear tires touched measuring force platform before the role, and compared with the landing gear load. The impact of peak load forward, avoid be included in the calculation of aircraft buffer system to absorb energy. At the same time, due to 
the simulation of the wing lift in advance, it is bound to reduce the initial speed when the test piece touches the measuring force platform. In the actual test, through appropriate to improve the test piece release altitude, utilizing increased potential energy offset consumed energy because of simulation of wing lift advance action. In the full scale aircraft drop test, the specific working condition should be iterated according to the sinking speed of test piece, in order to obtain the test piece rate of test requirements.

\section{Verification of Full Scale Aircraft Drop Test}

The validity of the method for simulating the lift of the wing is verified by the full scale aircraft drop test. Select the aircraft landing angle of 0 degrees, the sinking speed of $1.55 \mathrm{~m} / \mathrm{s}$ operating conditions to verify the test, during the test, the aircraft sinking speed, airplane attitude and wing lift size and time are collected.

The wing lift device and lift force applied method are applied to the full scale aircraft drop test. And the measured data are normalized, the simulated wing lift and landing gear ground load measured in the test are shown in Figure 12. The sinking speed of the aircraft gravity center measured in the test is shown in figure 13.

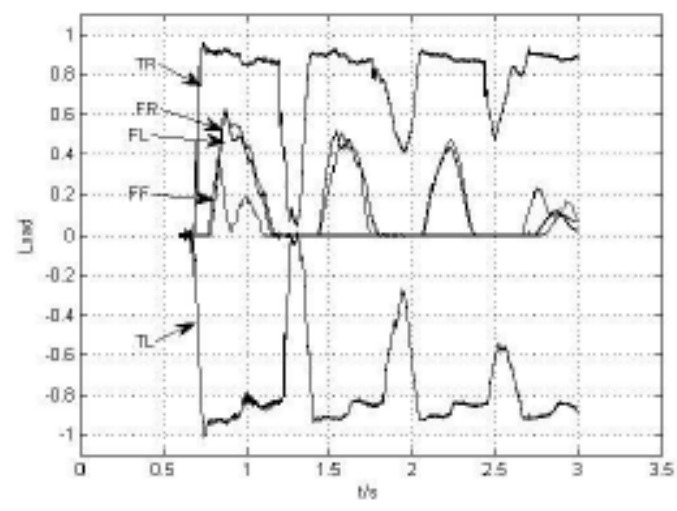

Figure 12. The typical working condition test curve

In the aspect of wing lift size applied, in fig12, TL and TR are the left and right wing lift, FF is the loads of the front landing gear, FL is the load of the left side of the landing gear, FR is the load of the right side landing gear. From the test curve we can see, simulating wing lift in the landing gear touches the load platform act in aircraft wing before touching the load platform, which effectively avoid the shock due to the sudden effect of wing lift generated by the sudden increase in load segment, making the whole process of the landing gear contacts with test platform, compression force of wing lift is basically stable. In the dynamic test, founding the actual value of wing lift is slightly greater than the theoretical value. Since the friction between the cylinder and the piston to be reversed during the anti-stroke of the landing gear, the actual value of lift appears step decreases. In the case of consistent height, the step value is about $2 \mathrm{kN}$, with the theoretical error of $2.67 \%$. The theoretical power of the aircraft is $47.44 \mathrm{~kJ}$. The power absorbed by the simulated wing lift and the absorption capacity of the buffer system are integrated according to (6) (7).

$$
A_{T}=\int_{Y_{c S}}^{Y_{c \max }} \sum_{i=1}^{2} T_{i} d y_{c}
$$

$\mathrm{A}_{\mathrm{T}}$ is the energy absorbed by the wing lift simulator. $\mathrm{Y}_{\mathrm{cs}}$ is the center of gravity displacement of the airplane when lift is applied. $\mathrm{Y}_{\mathrm{cmax}}$ is the maximum displacement of the center of gravity, and $\mathrm{T}$ is the wing lift measured by the load cell.

$$
A_{F}=\int_{0}^{Y_{c \max }} \sum_{i=1}^{3} F_{i} d y_{c}
$$


$A_{F}$ is the energy absorbed by the buffer system. $Y_{c m a x}$ is the maximum displacement of the center of gravity, and $\mathrm{F}$ is the landing load that each landing gear is subjected. The absorption capacity of the aircraft buffer system and the wing lift capacity are shown in Table 3.

Table 3. Energy of full scale aircraft drop test

\begin{tabular}{|c|c|c|c|}
\hline Total energy & $\begin{array}{c}\text { Energy absorbed by the wing } \\
\text { lift simulator }\end{array}$ & $\begin{array}{c}\text { Energy absorbed by the } \\
\text { buffer system }\end{array}$ & Error \\
\hline $\mathrm{kJ}$ & $\mathrm{kJ}$ & $\mathrm{kJ}$ & $\%$ \\
\hline 47.44 & 33.59 & 13.22 & 1.33 \\
\hline
\end{tabular}

It can be see from Table 3, at the sinking rate of $1.55 \mathrm{~m} / \mathrm{s}$, the total power delivered in the full scale aircraft drop test is converted into the energy absorbed by the wing lift and the energy absorbed by the aircraft buffer system. The error between the calculated energy and the tested energy is $1.33 \%$.

On the impact of aircraft landing attitude, it can be seen from the load curve, the initial state of the horizontal attitude, the aircraft nose gear, left and right main landing gear test load applied at the same time. After simulating the lift of the wing, the landing gear touches the force platform and maintains the horizontal attitude. Simulating the lift of the wing does not change the original falling attitude of the aircraft.

Aircraft sinking speed impact can be seen from Figure 13, the sinking velocity at the center of gravity of the airplane reaches $1.55 \mathrm{~m} / \mathrm{s}$, which proves that it is feasible to increase the altitude of the aircraft and offset the implementation of the method by simulating the lift action of the wing lift.

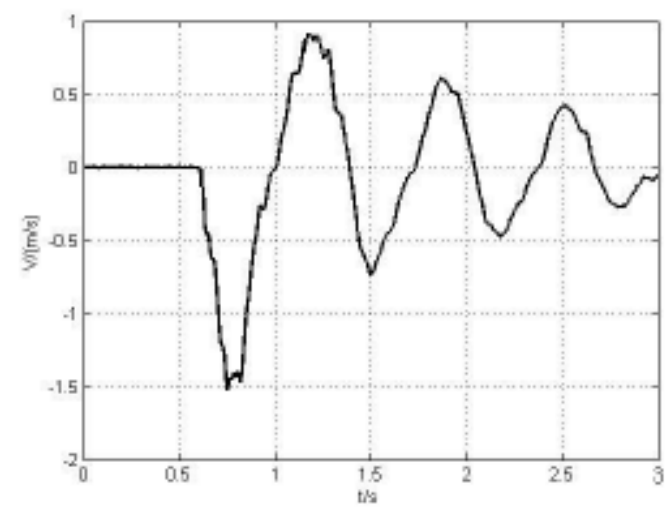

Figure 13. Aircraft sinking velocity curve

\section{Summary}

In this paper, the method of simulating the lift loading of the wings is proposed。 It is realized by two air medium cylinders acting on the wing on both sides of the aircraft respectively. The size of the wing lift is achieved by adjusting the pressure of the gas tank. The lift action point is located at the center of gravity of the aircraft, perpendicular to the fuselage section, and avoids the influence of the wing lift on the landing attitude of the aircraft. And Lift force is applied before the landing gear touches the platform to avoid the influences of the impact load on the landing load.

Wing lift simulator with integrated air cylinder and air tank is designed. And the influence of the design parameters on the output lift force is analyzed. Both static and dynamic verification of the lift simulator are carried out respectively. Static verification result shows that the theoretical value and the measured value error is less than $3 \%$. And through the dynamic verification, the causes and solutions of the impact peak load are analyzed.

Through the full scale aircraft drop test, From the simulation of wing lift size, and influence of landing attitude and sinking speed of aircraft the applicability of the wing lift loading method and the lift simulation device to the full scale aircraft drop test is verified systematically. The test results 
shows that the applying method of device and method can meet the load requirements of the full scale aircraft drop test.

\section{References}

1. Committee of Aircraft Design Manual.Aircraft design manual book 14:taking-0ff and landing devices design[M].Beijing:Aviation Industry Press:95-102,(2002)

2. Liu Ruichen, Su Kaixin, Shao Yongqi, etal. Strength design guide of aircraft landinggear[M]. Sichuan: Sichuan Science and Technology Press. (1989)

3. Wei xiaohui, Nie hong. New method of attenuating landing impact force on carrier-based aircraft landing gear[J]. Acta Aeronautica et Astronautica Sinica, 28(2): 324-327(2007)

4. NIE H, Peng Y M,Wei X H, et al. Overview of carrier-based aircraft arrested deck-landing dynamics [J]. Acta Aeronautica et Astronautica Sinica, 35(1): 1-12(2013)

5. WANG Q S. Critical technologies in carrier-based aircraft design and development[J]. Aircraft Design, 6-10(2005).

6. Ryberg E S. The influence of ship configuration on the design of the joint strike fighter, ADA399988[R]. Arlington, VA: Joint Strike Fighter Program Office (2002)

7. MIL-A-8863C(AS). Military specification airplane strength and rigidity ground loads for navy acquired airplanes[S]. (1960)

8. MIL-A-8867C(AS). Military specification airplane strength and rigidity ground loads Tests[S]. (1998)

9. JSSG-2006. Airplane structures[S]. Naval Air Systems Command. (1998)

10. Duan Pingping. Investigation on dynamic performance analysis for carrier-based aircraft during landing process[D]. NanJing: College of Aerospace Engineering, Nanjing University of Aeronautics and Astronautics (2012)

11. WEI X H, Nie H. Analysis of landing impact force of aircraft landing gears based on the conception of landing area[J]. Acta Aeronautica et Astronautica Sinica, 26(1): 8-12(2005)

12. Zhu Ling. Research on land-gear load carrier-based arresting[D]. NanJing: College of Aerospace Engineering, Nanjing University of Aeronautics and Astronautics(2009)

13. Qi Peiqian, Shi Weiqi, Qi Zhiming. Wing Lift Simulation in Landing-gear Drop Test[J]. Mechanical Science And Technology. 21(S1):36-37(2002)

14. Yao niankui Zhou dong. Full-Scale Drop Test of Fixed-Wing Carrier-Based Aircraft[J]. Aircraft Design, 34(4):31-36(2014)

15. YAO N K, Sui F C, Wang C B. Free flight engagement condition of carrier-based aircraft[J]. Aircraft Design, 31(6):10-15(2011) 